\title{
Optimization of REMS Program Compliance in a Large Academic Health System
}

\author{
Audrey B. Kostrzewa, PharmD, MPH, BCPS \\ Froedtert \& the Medical College of Wisconsin (F\&MCW) \& Concordia University Wisconsin (CUW)
}

\begin{abstract}
Ensuring compliance with all applicable Risk Evaluation and Mitigation Strategies (REMS) programs within a health system is challenging. These FDA-mandated programs are numerous, unique, and ever-changing. Actions require several stakeholders and moving parts. In addition, the effectiveness and impact of these programs has been challenged and is not always clear, which hampers buy-in and therefore compliance.

F\&MCW is a health network in southeastern Wisconsin that includes five hospitals and almost 40 clinics. Since 2014, several systemwide approaches to medication use, including formulary alignment and REMS program optimization, have taken place with an overarching goal of providing efficient, safe, and consistent care for the patients and populations served.

This manuscript describes the steps that took place over the past six years or so related to REMS program optimization. It also offers practical tips for other health systems based on lessons learned through this one institution's experiences.
\end{abstract}

Keywords: Risk Evaluation and Mitigation Strategies, REMS, Medication Safety, Health System, FDA

\section{The Background and Challenges with Risk Evaluation and Mitigation Strategies (REMS) Programs}

Anyone who has ever tried to implement a Risk Evaluation and Mitigation Strategies (REMS) program in their pharmacy or health system, let alone multiple, knows how challenging it can be. ${ }^{1-4}$ There are many various programs that are all different and ever-changing. The actions required can involve several stakeholders and moving parts. In addition, it can be hard to clearly see the connection to effectiveness and impact of these programs $^{4-8}$, which hampers buy-in and therefore compliance. 9,10

The Food and Drug Administration (FDA) Amendments Act in 2007 gave the FDA authority to require drug manufacturers to provide additional information, or REMS, to ensure the benefits of using a medication would outweigh the risks, as the FDA deemed appropriate. ${ }^{2,11-13}$ These programs vary by medication, but can include any of up to four components: medication guide, elements to assure safe use (ETASU), implementation plan, and communication plan. Health care professionals, institutions, and pharmacies are required to carry out certain actions in these programs, and historically it has been very difficult to ensure compliance with each of these unique programs. $^{14}$

In response to the operational challenges, the FDA implemented the REMS Integration Initiative in 2011, and has been working on making improvements ever since. ${ }^{15}$ After soliciting stakeholder feedback, the FDA developed four priority

\section{Corresponding author:}

Audrey B. Kostrzewa, PharmD, MPH, BCPS

Froedtert \& the Medical College of Wisconsin (F\&MCW) \&

Concordia University Wisconsin (CUW)

Phone: (262) 243-2750; Email: audrey.kostrzewa@cuw.edu projects, one of which was to provide a central source of REMS information for practice settings through an improved website. The REMS website has been drastically improved, making it much more user friendly. ${ }^{11}$ In July 2017, the FDA also released the REMS Platform Standards Initiative: Needs Assessment ${ }^{16}$, providing an opportunity for stakeholders to evaluate their own current processes.

Understanding the origin and significance of REMS programs, and what the FDA is doing to improve information and processes on their end is important, but practically carrying out the requirements for all relevant REMS programs on a system level is still challenging. There are many players and moving parts; having one person or one group of people primarily responsible for overseeing REMS processes can promote efficiency and success.

\footnotetext{
One Innovative Approach at a Large Academic Medical Center Froedtert \& the Medical College of Wisconsin (F\&MCW) $\mathrm{F} \& M C W$ is a health network in southeastern Wisconsin that includes five hospitals and almost 40 clinics. ${ }^{17}$ It is home to eastern Wisconsin's only academic medical center and Level I Trauma Center at Froedtert Hospital in Milwaukee. The Center for Medication Utilization (CMU) team is a group of pharmacists who help optimize how medications are used in the F\&MCW health network. In 2014, when there were three hospitals in the network, all three hospital formularies were aligned to one system-wide formulary. Since then, a clinic-based formulary was established, and in 2020, the entire health system, inpatient and outpatient, was aligned to one system-wide formulary. During the process of aligning formularies, other system-wide approaches to medication use have happened concurrently, such as REMS program optimization. All of this work has had the same over-arching goal of providing efficient, safe, and consistent care for the patients and populations served.
} 
Figure 1. System-Wide Stepwise Approach to REMS Programs at F\&MCW

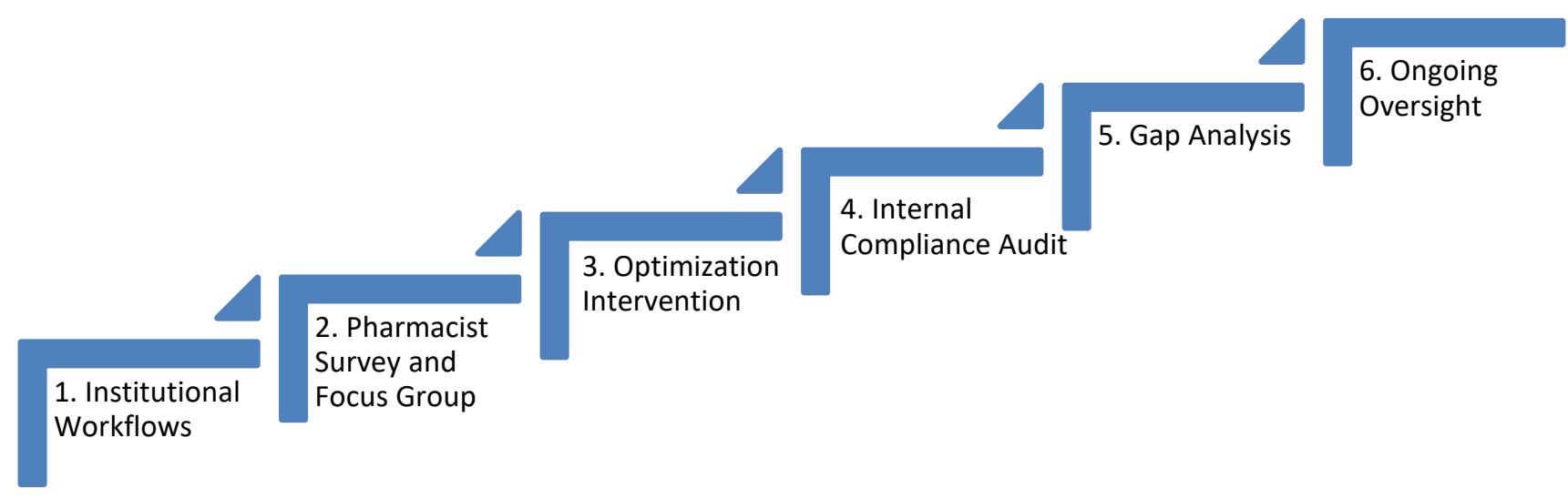

\section{Step 1: Institutional Workflows}

Several years ago, members of the $\mathrm{CMU}$ team at $\mathrm{F} \& \mathrm{MCW}$ developed REMS workflows to assist frontline staff in knowing what the requirements for each of these programs are, especially as it relates to F\&MCW. The workflows were posted on the intranet and there was some integration with the electronic health record (EHR) system to alert end-users to the various requirements. This included instructions and questions within the medication order itself, a priority flag on a summary view to alert the pharmacist when a REMS medication required a lab test, and a link to the medication guide within the medication administration record (MAR). Education of staff involved inpatient and outpatient staff meetings and an EHR education document. Ongoing maintenance of these workflows is overseen by the Medications Warnings and Alerts (MWA) workgroup, a system-wide pharmacy group who meets monthly to review new safety information from FDA, Institute for Safe Medication Practices (ISMP), etc. Then, after a year or so, a REMS program audit and some anecdotal evidence suggested that staff members were not very familiar with REMS programs and the resources available to them at F\&MCW.

\section{Step 2: Pharmacist Survey and Focus Group}

The objective of the survey and focus group was to evaluate, identify gaps, and make improvements to current REMS process in order to optimize compliance, prepare for potential audits, and improve patient safety. First, a report was run from the EHR system to identify REMS medications administered in the health system ( 3 hospitals) between July 1, 2016 and June 30, 2017, including stratification by hospital and floor. Pharmacists were then identified based on primary work location with highest historical usage of one of the inpatient REMS medications (clozapine) to participate in a focus group in October 2017. Six pharmacists, including at least one pharmacist from each of the three hospitals in the health system, were identified and contacted to participate in the focus group. The goal of the focus group was to find out more information about current logistics and compliance, particularly with the clozapine REMS program, as well as suggestions for future directions. Results were used to compile the survey. The 9-question survey was sent out to all pharmacists in November 2017 via SurveyMonkey@. Pharmacists were asked to selfidentify themselves as working on a floor with highest REMS medication utilization (identified in the email) within the past year. Forty-seven pharmacists completed the survey with all three hospitals represented. Two of the nine survey question results are outlined below in Figure 1. These two questions illustrate that in general, pharmacists were not very confident when it comes to REMS programs and they usually rely on the EHR to tell them whether a medication has a REMS program. The survey also asked about current documentation practices. The majority of pharmacists (33/47 or $70.2 \%$ ) did document about REMS somewhere in the EHR, but when asked an openended question about where, there were several different locations identified. The documentation practices were not consistent and not queryable. Results of the survey were analyzed to determine current state and optimal interventions for REMS compliance. 
Figure 1: Pre-intervention Survey

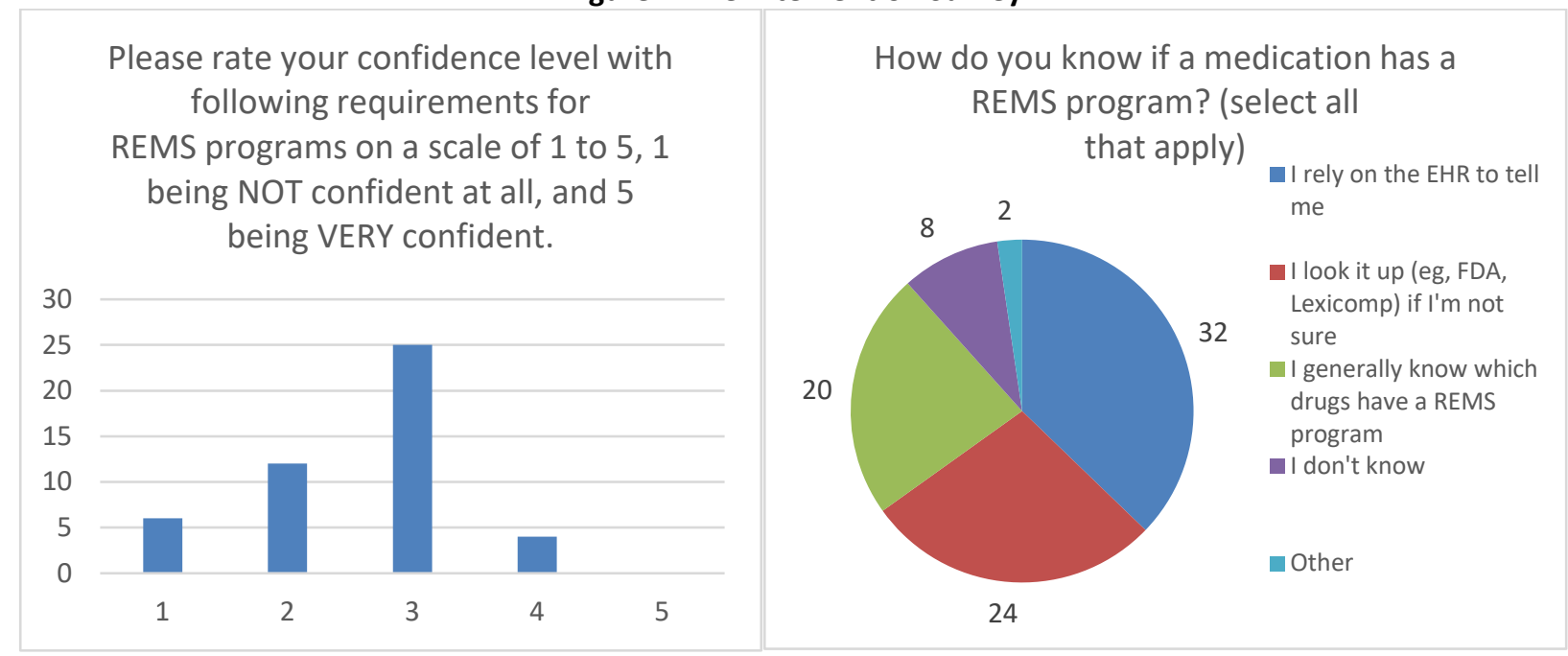

\section{Step 3: Optimization Intervention}

Next, a plan for intervention was developed. The goals of any potential intervention were to: 1) prompt staff to document appropriately for each REMS program (i.e., passive), 2) not to egregiously interrupt workflows or cause a lot of extra work for anyone involved, and 3 ) be queryable in the EHR for future internal or external audits. Based on the survey results and the goals listed previously, the following interventions were determined to be most beneficial:

- Banner (shows up on a main page in EHR when a patient is on a REMS medication) that prompts pharmacist to complete a note (see below)

- Note template (walks pharmacist through all necessary information for each unique REMS program)

There were six medications on the health system formulary that required action to be taken on the inpatient side: alvimopan (Entereg); ambrisentan (Letairis); bosentan (Tracleer); clozapine (Clozaril); macitentan (Opsumit); and riociguat (Adempas). The note template was built based on these six REMS programs and workflows. This type of documentation is not required by REMS programs, but would help accomplish the internal goals listed above. The template walks the user through information and requirements for each unique program, populating different information based on responses the user gives. For example, the first item the user selects is the name of the medication from a drop-down menu. Based on the medication chosen, more information populates for the unique REMS program. The note was also built to automatically populate some basic patient information directly from the EHR. Overall, the intervention took approximately six months to develop and implement, from the pre-intervention survey to the go-live date, including staff education through in person staff meetings, email, and the EHR.

\section{Step 4: Internal Compliance Audit}

After the intervention went live, one year of data was collected to observe compliance. From August 2018 (first month after golive) to July 2019 (12 months), there were a total of 777 admissions for 545 unique patients within the health system with an inpatient REMS medication given based on the MAR. Of the 777 admissions, 318 had at least one note template completed (56 admissions had more than one), resulting in $40.93 \%$ compliance. Of note, this refers to compliance with completing the note, which is a surrogate for REMS compliance. There may be instances where pharmacists are using past methods to document which would not be queryable and therefore not captured in this audit. One example of likely REMS program compliance without the note being completed is alvimopan, which accounts for $425 / 777$ (54.7\%) of the admissions. The main REMS criteria for this medication is to ensure that no more than 15 doses are given to each patient. This is mitigated in other ways through the EHR such as within the medication order itself. There was a large medication use evaluation (MUE) completed on this medication more recently than when this internal REMS audit happened. To provide a more robust audit, a detailed review of each medical record would likely need to be performed.

\section{Step 5: Gap Analysis}

Concurrently with the inpatient-focused optimization project, a gap analysis of all 74 (as of 2018) FDA-mandated REMS programs and available institution-specific workflows (18 as of 2018) was performed. Data was gathered from EHR prescriptions, outpatient pharmacy dispensing, and the formulary (to account for clinic administrations). Of the 74 FDA 
programs, 47 had requirements for medication guide and/or ETASU which are the two REMS components that institutions need to act on. Of the 47 remaining, 18 already had workflows, 16 were not on the formulary, and four were for inpatient use only, leaving 9 potential outpatient workflows. Five of the 9 had med guide dispensing in the outpatient pharmacies only. These print off automatically in the pharmacy, so no additional guidance on workflow is necessary. Overall, there were four outpatient workflows identified as needing to be developed as a result of the gap analysis, which were created and updated as appropriate.

\section{Step 6: Ongoing Oversight}

Ongoing monthly surveillance of changes to REMS programs happens through a system-wide safety-focused group called Meds, Warnings, and Alerts (MWA) Workgroup. The group meets monthly to review national safety information from the ISMP, FDA, etc. One of the standing agenda items is REMS programs. This monthly review prompts updates to workflows to ensure the most updated and accurate information is available to staff.

\section{Future Directions and Lessons Learned}

Like any quality improvement initiative, there were strengths and limitations learned throughout the process. Strengths include using one person/group to oversee, consistency with the templates, and sustainability through the MWA Workgroup. Limitations include trying to take on all possible REMS programs instead of focusing efforts, and not continuing to re-educate staff to ensure understanding and compliance.

Moving forward, the plan is to carve out outpatient pharmacyonly (non-formulary) REMS programs and delegate them to an outpatient pharmacy-focused committee. More work will need to be done to ensure that inpatient REMS note compliance is improved and that it serves as a realistic surrogate marker for REMS compliance. In addition, to improve sustainability further, other stakeholder pharmacists will be involved in the oversight and reviewing workflows and processes on a more pro-active, regular basis.

It has been several years and steps into this process. A lot has been learned and the hope is that by sharing one institution's experience, others may be successful. Here are a few practical tips recommended for others:

- Have one person or one group of people primarily responsible. This can be either for all REMS programs within an institution or by service line/location (e.g., oncology, infusion clinic). For F\&MCW, select members of the CMU team oversee all REMS programs and delegate certain responsibilities to service line/location stakeholders as necessary. Streamlining responsibilities and communication promotes efficiency.
- Scope what will and will not be overseen. It can be easy to get overwhelmed with the volume of REMS programs and number of changes. It is ideal to draw a line in the sand. Examples of how to scope include formulary products only, inpatient (or outpatient) only, required action only (vs "suggested" or "optional"). From experience, the inpatient/outpatient and required/suggested lines can be blurry with some REMS programs.

- Communicate with and solicit feedback from frontline staff. They are the eyes and ears, and compliance with REMS programs relies heavily on frontline staff to understand and follow-through on requirements. Getting their buy-in and feedback is crucial.

- Do not "set it and forget it". Things get outdated quickly and staff turns over, so having a plan for sustainability, both for education and for responding to changes with REMS programs is really important.

REMS programs and the FDA have come a long way since 2007. Although they may have been challenging to comply with, and continue to offer some growing pains, sharing experiences and best practices can help alleviate at least some of the burden. Ultimately, REMS programs exist to ensure safe and effective access to medication, which is the ultimate, shared goal.

Acknowledgements: The management of REMS programs at F\&MCW has gone through several steps over the years and the following individuals should be acknowledged for their contributions: Julie Karpinski, Katie Kotz, Rachel Parry, Olivia Lemanski, and Taylor Mancuso.

Funding/Support: None

Conflicts of Interest: None

Treatment of Human Subjects: The project was reviewed and approved by the F\&MCW Pharmacy Department Research Committee. It was approved as a quality improvement project and requirement for IRB review was waived. 


\section{References}

1. Childs L, Alexander E, Duong M-T. Risk evaluation and mitigation strategies: Assessment of a medical center's policies and procedures. American Journal of Health-System Pharmacy. 2012;69(10):885-889. doi:10.2146/ajhp110356

2. Nelson LS, Loh M, Perrone J. Assuring Safety of Inherently Unsafe Medications: the FDA Risk Evaluation and Mitigation Strategies. Journal of Medical Toxicology. 2014;10(2):165-172. doi:10.1007/s13181-013-0374-z

3. Reiss SM. APhA 2011 REMS white paper: Summary of the REMS stakeholder meeting on improving program design and implementation. American Pharmacists Association. Journal of the American Pharmacists Association. 2011;51(3):340-358. doi:10.1331/JAPhA.2011.11519

4. Smith MY, Seligman PJ. New Opportunities for Integrating Drug Safety Risk Management Programs Into the Health Care System: Bridging the Divide. JAMA. 2015;314(17):1793. doi:10.1001/jama.2015.11871

5. Cronin M, Berger S, Seligman PJ. Risk Evaluation and Mitigation Strategies With Elements to Assure Safe Use: Alignment of the Goals With the Tools to Manage Risk. Therapeutic Innovation \& Regulatory Science. 2014;48(6):724-733. doi:10.1177/2168479014527749

6. Boudes PF. Risk Evaluation and Mitigation Strategies (REMSs): Are They Improving Drug Safety? A Critical Review of REMSs Requiring Elements to Assure Safe Use (ETASU). Drugs in R\&D. 2017;17(2):245-254. doi:10.1007/s40268-017-0175-y

7. Toyserkani GA, Huynh L, Morrato EH. Adaptation for Regulatory Application: A Content Analysis of FDA Risk Evaluation and Mitigation Strategies Assessment Plans (2014-2018) Using RE-AIM. Frontiers in Public Health. 2020;8. doi:10.3389/fpubh.2020.00043

8. Guadamuz JS, Qato DM, Alexander GC. Use of Risk Evaluation and Mitigation Strategies by the US Food and Drug Administration, 2008-2019. JAMA. 2020;324(3):299. doi:10.1001/jama.2020.6611
9. Wu J, Juhaeri J. The US Food and Drug Administration's Risk Evaluation and Mitigation Strategy (REMS) Program - Current Status and Future Direction. Clinical Therapeutics. 2016;38(12):25262532. doi:10.1016/j.clinthera.2016.11.007

10. Prokes M, Root A. A Retrospective Analysis of Adherence to Risk Evaluation and Mitigation Strategies Requirements for Pulmonary Arterial Hypertension Drugs. Hospital Pharmacy. 2019;54(5):309-313. doi:10.1177/0018578718791509

11. Approved Risk Evaluation and Mitigation Strategies (REMS). U.S. Food \& Drug Administration Accessed November 18, 2020. https://www.accessdata.fda.gov/scripts/cder/rems/

12. Lippmann E. Risk Evaluation and Mitigation Strategies (REMS). Presented at the: Accessed November 18, 2020. https://www.fda.gov/media/105565/download

13. Hollingsworth $\mathrm{K}$, Toscani M. Risk Evaluation Mitigation Strategies: The Evolution of Risk Management Policy. Population Health Management. 2013;16(2):132-137. doi:10.1089/pop.2012.0021

14. Stubbings J, Joshi RA, Hoffman JM. Risk evaluation and mitigation strategies: Challenges and opportunities for health-system pharmacists. American Journal of Health-System Pharmacy. 2010;67(18):1547-1554. doi:10.2146/ajhp090640

15. REMS Integration Initiative.https://www.fda.gov/industry/prescriptiondrug-user-fee-amendments/rems-integrationinitiative. Published February 2, 2018. Accessed November 18, 2020.

16. REMS Platform Standards Initiative: Needs Assessment. U.S. Food \& Drug Administration Accessed November 18, 2020. https://www.fda.gov/media/106442/download

17. Froedtert \& the Medical College of Wisconsin. Published online 2020. Accessed November 18, 2020. https://www.froedtert.com/ 